\title{
等离子体光催化剂在有机合成中的应用研究进展
}

\author{
王 春* 高书涛周 欣 吴秋华 教彩娜 王 志* \\ (河北农业大学理学院 保定 071001)
}

\begin{abstract}
摘要 光催化有机合成作为光催化领域的一个前沿方向, 越来越受到人们的广泛关注. 基于金属表面等离子体共振效 应的等离子体光催化剂作为一类新型可见光响应的光催化剂, 已成功用于催化醇氧化、烯烃环氧化、硝基还原、碳碳 偶联、苯着基化等诸多重要的有机反应, 在利用太阳能方面显示出巨大的应用潜力. 总结了等离子体光催化剂在有机 合成中的最新研究进展, 并对其发展趋势进行了展望.
\end{abstract}

关键词 光催化; 等离子体; 有机合成; 催化; 综述

\section{Research Progress of Plasmonic Photocatalyst in Organic Synthesis}

\author{
Wang, Chun* Gao, Shutao Zhou, Xin Wu, Qiuhua Jiao, Caina Wang, Zhi* \\ (College of Science, Agricultural University of Hebei, Baoding 071001)
}

\begin{abstract}
Photocatalytic chemical transformations are a hot topic in photocatalysis field and have received significant attention in recent years. Plasmonic photocatalysts based on metal surface plasmon resonance are novel visible light response photocatalysts, which have been applied in the catalysis of various important organic reactions, such as aerobic oxidation of alcohols, epoxidation of alkene, reduction of nitro-compounds, $\mathrm{C}-\mathrm{C}$ coupling, and oxidation of benzene to phenol. Herein the recent progresses in selective organic synthesis catalyzed by plasmonic photocatalysts are reviewed and a perspective on the development trend is given.
\end{abstract}

Keywords photocatalyst; plasmon; organic synthesis; catalysis; review

随着经济的迅猛发展, 环境污染和能源危机已经成 为人类社会所面临的重要挑战. 所以，发展可再生能 源、消除化学工业对环境的有害影响, 成为未来化学研 究中的热点问题. 光催化技术具有洁净、安全、操作简 单、无二次污染等优点, 是解决人们所面临的能源和环 境问题的一种有效途径. 光催化技术在分解水制氢气、 环境污染物降解、光电转化、有机合成等领域的应用引 发了广泛的关注 ${ }^{[1]}$.

作为一种取之不尽、廉价易得的能源, 太阳光在驱 动环境友好的有机转化中具有很大的应用潜力. 众所周 知, 传统的有机合成不仅步骤繁琐, 一些反应还需要在 高温高压下才能进行, 而光催化反应体系通常在简单温 和的条件下即可进行, 避免了在传统有机合成中所使用 的复杂步骤和苛刻条件, 使由此带来的环境和能源问题 得到有效解决, 是实现有机合成绿色化的一种新途径. 到目前为止, 人们研究最多光催化材料是金属氧化物半
导体光催化材料. 有关半导体光催化剂催化的选择性有 机转化已有较多报道 ${ }^{[2]}$, 然而, 这些光催化剂需要紫外 光激发才能显示催化活性, 而紫外光诱导的化学反应中 含有自由基中间体，使反应的选择性不尽令人满意. 而 太阳光主要由可见光组成, 开发可见光响应的光催化 剂, 以在有机转化反应中有效利用太阳能成为人们关注 的焦点 ${ }^{[3]}$. 当前国内外在这方面的研究工作主要集中在 以下两个方面: (1)对半导体光催化材料进行改性和探索 具有可见光响应的新型半导体材料体系来扩展现有光 催化材料的光响应范围; (2)使用具有可见光吸收的等离 子体光催化剂.

等离子体光催化剂是基于金属表面等离子体共振 效应的一类对可见光有良好响应的新型光催化剂 ${ }^{[4]}$. 金 属中的表面等离子体共振是描述其导带电子在电磁场 作用下集体振荡的一个物理概念. 由入射光和金属表面 的自由电子相互作用而产生. 具有一定频率的入射光波

\footnotetext{
*E-mail: chunwang69@126.com; wangzhi@hebau.edu.cn

Received May 12, 2014; revised June 25, 2014; published online July 3, 2014.

Project supported by the Natural Science Foundation of Hebei Province (No. B2011204051) and the Innovation Research Program of Department of Education of Hebei for Hebei Provincial Universities (No. LJRC009).

河北省自然科学基金(No. B2011204051)和河北省高等学校创新团队领军人才培育计划(No. LJRC009)资助项目.
} 
可以诱导金属的外层自由电子产生极化, 进而诱导其表 面表现出净电荷, 该净电荷相当于一个新建电场, 于是 就会产生静电回复力, 其静电回复力可以使电子发生偶 极振荡, 当入射光的频率与电子的集体振荡频率一致 时, 则可以发生耦合共振, 这种共振在宏观上就表现为 金属对光的吸收, 这就是通常所谓的表面等离子体共振 (Surface Plasmon Resonance, 简称 SPR) ${ }^{[4 b]}$.

贵金属特别是金、银、铜等纳米粒子在可见区域有 较强的吸收, 其表面等离子体共振吸收光谱的最大吸收 波长分别为 $530 、 400 、 580 \mathrm{~nm}$. 因此, 利用金、银、铜 的 SPR 效应可以在可见光的照射下调控化学转化 ${ }^{[2 b]}$. 尽管独立的金属等离子体纳米材料能够在可见光的照 射下催化有机物转化, 但它们的稳定性较差, 因此在实 际应用中, 大多数等离子光催化剂负载到金属氧化物 上 ${ }^{[3 \mathrm{a}]}$, 如可被还原的金属氧化物 $\mathrm{CeO}_{2}$ 以及惰性载体 $\mathrm{ZrO}_{2} 、 \mathrm{Al}_{2} \mathrm{O}_{3} 、 \mathrm{SiO}_{2}$ 等, 而 $\mathrm{TiO}_{2}$ 因其耐光腐蚀、耐酸碱 性好、化学性质稳定、对生物无毒、成本低、可循环利 用等优点成为目前应用最广泛的光催化材料, 是最受欢 迎的金属氧化物载体. $\mathrm{TiO}_{2}$ 的导带能帮助电子转移到电 子受体中, 如氧气, 这有利于选择性需氧氧化.

等离子体光催化剂在有机合成领域具有巨大的发 展潜力和广阔的应用前景, 已引起了广大有机化学家的 广泛关注, 并已取得了一些新的研究成果. 本文主要对 近年来国内外有关等离子体光催化剂在有机转化反应 中的应用进行简要评述, 并对存在问题及未来发展趋势 进行了总结和展望.

\section{1 等离子体光催化剂的光催化机理}

等离子光催化剂催化有机转化的机理目前还没有 完全一致的认识. 大多数等离子光催化剂是将贵金属负 载到金属氧化物上, 一般认为此类催化剂的作用机理可 用 Scheme 1 表示 ${ }^{[3 a, 4 a]}$.

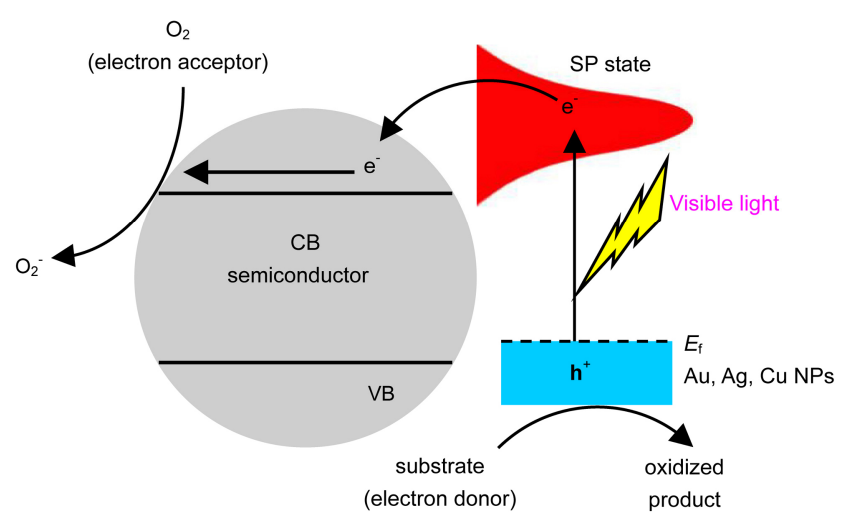

Scheme 1

在可见光的照射下，等离子金属如金、银、铜等纳
米粒子吸收光能后使得位于费米能级 $E_{\mathrm{f}}$ 附近的电子被 激发成表面等离子体(SP)状态，与低于 $E_{\mathrm{f}}$ 的带正电荷的 空穴 $\left(\mathrm{h}^{+}\right)$分离. 由于等离子体纳米粒子被负载在金属氧 化物上，因此电子将会转移到金属氧化物的导带(CB) 中，并且最终转移到电子受体如氧气中，同时，留下的 带正电的空穴将会被有机底物淬灭, 完成光催化循环. 为了全面的理解等离子体光催化剂的催化机理，等离子 体纳米粒子和金属氧化物载体的性能都应该考虑. 金属 氧化物载体除了能稳定等离子体纳米粒子和帮助转移 电子的作用外，也具有酸、碱、配位等功能，这可能对 多相反应过程有促进作用. 另外, 金属氧化物负载的 金、银、铜纳米粒子是良好的异质结光催化剂，有利于 热诱导的氧化还原反应的进行，而氧化还原反应的发生 又能进一步促进等离子体光催化的进行.

\section{2 等离子体光催化剂在有机合成中的应用}

\section{1 醇氧化反应}

醇类化合物的氧化为相应的羰基化合物的反应是 有机合成中最常用的反应，因为羰基化合物是制造药 物、维生素及香料的重要前体物，所以该反应在工业生 产中具有十分重要的作用. 通常羰基化合物的合成需要 高温高压条件, 且使用的氧化剂是具有毒性或腐蚀性的 强氧化剂如重铬酸钾、高锰酸钾等. 利用光催化反应可 避免上述问题, Shiraishi 等 ${ }^{[5]}$ 将直径小于 $5 \mathrm{~nm}$ 的金纳米 粒子负载在锐钛矿型/金红石型 $\mathrm{TiO}_{2}$ 混晶结构的界面上, 制备了 $\mathrm{Au} / \mathrm{TiO}_{2}$ 等离子光催化剂, 在可见光 $(\lambda>450 \mathrm{~nm})$ 的照射下，成功地催化芳香醇类在室温下发生氧化反 应, 反应效率高且选择性好(Eq. 1). 催化剂 $\mathrm{Au} / \mathrm{TiO}_{2}$ 是 以 $\mathrm{H}_{4} \mathrm{AuCl}_{4}$ 和 $\mathrm{TiO}_{2}$ 为原料, 通过沉积沉淀法合成. 实验 发现不同晶型的 $\mathrm{TiO}_{2}$ (即锐钣矿型、金红石型、Degussa P25)作为金纳米粒子的载体时催化效果不同, Degussa P25 $\mathrm{TiO}_{2}$ 作为载体催化活性最高. 研究还表明光照可大 大提高催化活性, 产率是非光照条件下的 4 倍多. 另外 金粒子的大小及在 $\mathrm{TiO}_{2}$ 上附着的位置对催化效率也有 很大影响，小于 $5 \mathrm{~nm}$ 的金纳米粒子负载在锐钛矿型/金 红石 $\mathrm{TiO}_{2}$ 混晶的界面上时催化效率显著提高.

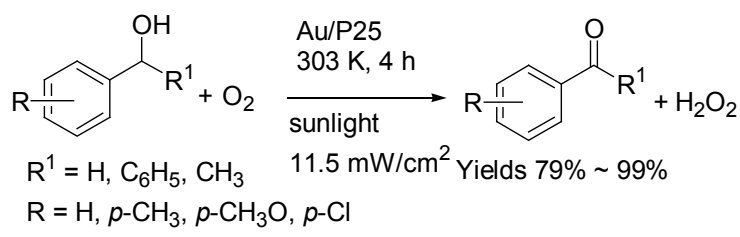

Tada 等 ${ }^{[6]}$ 发现, 在可见光的照射下, 在 $\mathrm{Au} / \mathrm{TiO}_{2}$ 体 系中加入表面活性剂三甲基十八烷基氯化铵 $\left(\mathrm{C}_{18} \mathrm{TAC}\right)$ 时，可有效催化水相中醇类化合物氧化为羰基化合物的 
反应，反应具有很高的选择性( $>99 \%)$. 其中 $\mathrm{Au} / \mathrm{TiO}_{2}$ 是 由 $\mathrm{H}_{4} \mathrm{AuCl}_{4}$ 和 $\mathrm{TiO}_{2}$ 采用沉积沉淀法合成的, 当阳离子型 表面活性剂 $\mathrm{C}_{18} \mathrm{TAC}$ 添加到该光催化体系时, 反应产率 一般会提高 3.3 5.7 倍, 对某些个别化合物, 产率可增 大 29.6 倍. 通过动力学研究发现, 除了 4-HO$\mathrm{C}_{6} \mathrm{H}_{4} \mathrm{CH}_{2} \mathrm{OH}$ 外, 其他醇类氧化形成相应羰基化合物呈 Hammett 线性相关.

据报道, $\mathrm{CeO}_{2}$ 作为一种可还原的金属氧化物, 可以 代替 $\mathrm{TiO}_{2}$ 作为金纳米粒子的载体. Kominami 研究组 ${ }^{[7]}$ 以柠檬酸作为还原剂, 在 $\mathrm{CeO}_{2}$ 表面还原 $\mathrm{H}_{4} \mathrm{AuCl}_{4}$ 制备 了 $\mathrm{Au} / \mathrm{CeO}_{2}$ 等离子光催化剂, 所获得的 $\mathrm{Au} / \mathrm{CeO}_{2}$ 的最大 吸收波长在 $550 \mathrm{~nm}$ 左右, 与金纳米粒子的表面等离子 共振吸收一致. 实验表明, 在 $530 \mathrm{~nm}$ 的 LED 灯照射下 时, $\mathrm{Au} / \mathrm{CeO}_{2}$ 可以催化芳香醇在水相中被氧气选择性地 氧化成芳香醛, 反应几乎定量进行 (Eq. 2). 另外催化剂 的催化活性主要取决于负载在 $\mathrm{CeO}_{2}$ 上的金纳米粒子的 比表面积而不是金纳米粒子的总量. 实验表明当金纳米 粒子平均粒径为 $30 \mathrm{~nm}$ 时, 可作为一种非常有效的可见 光等离子光催化剂. Kominami 研究组 ${ }^{[8]}$ 还通过多步光化 学沉积的方法合成了 $\mathrm{Au} / \mathrm{CeO}_{2}$, 与单步合成法相比, 多 步合成法合成的金纳米粒子粒径大、催化活性高, 其催 化反应速率是单步合成法的 2 倍. 结果表明两种方法合 成的 $\mathrm{Au} / \mathrm{CeO}_{2}$ 催化醇类氧化都具有很高的选择性, 可选 择氧化对氨基苯甲醇为对氨基苯甲醛而不氧化氨基.

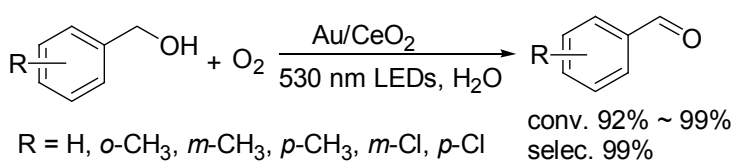

$\mathrm{Au} / \mathrm{CeO}_{2}$ 催化醇类氧化反应被认为涉及 $\mathrm{Au}-\mathrm{H}$ 中间 体, 最近 Maldotti 等 ${ }^{[9]}$ 采用自旋捕获与电子顺磁共振技 术考查了 $\mathrm{Au}-\mathrm{H}$ 中间体的作用途径. 实验证明该中间体 是控制 $\mathrm{Au}$ 纳米粒子催化氧化醇类反应的重要中间体, 且光照影响 Au-H 键的稳定性, 有助于该化学键的断裂, 从而恢复 $\mathrm{Au}$ 的催化活性, 所以光照能加速反应的进行.

金纳米粒子本身也能作为等离子体光催化剂, 例 如, 在 $530 \mathrm{~nm}$ LED 照射下, 金纳米粒子可催化双氧水 氧化芳香醇为相应的羰基化合物 ${ }^{[10]}$ (Eq. 3), 转化率可达 $95 \%$. 金纳米粒子可由氯金酸在苯甲酮和表面活性剂的 存在下通过光化学方法合成, 该法制备的金纳米粒子直 径大约 $15 \mathrm{~nm}$.

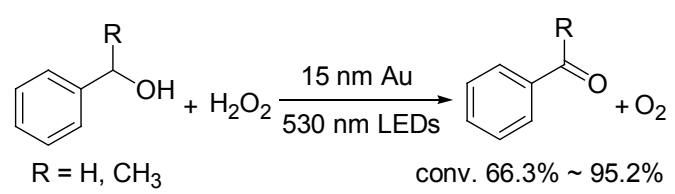

金-钯合金负载到 $\mathrm{ZrO}_{2}$ 上得到的 $\mathrm{Au}-\mathrm{Pd} / \mathrm{ZrO}_{2}{ }^{[11,12]}$ 或金-铜合金负载到 $\mathrm{TiO}_{2}$ 上得到的 $\mathrm{Au}-\mathrm{Cu} / \mathrm{TiO}_{2}{ }^{[13]}$, 在可 见光或太阳光的照射下可以提高氧气氧化苯甲醇的反 应活性. 对于 $\mathrm{Au}-\mathrm{Pd} / \mathrm{ZrO}_{2}$ 催化体系, $\mathrm{Au} 、 \mathrm{Pd}$ 的摩尔比 决定了纳米粒子表面钯位点的分布情况及电子的不均 匀性, 从而对催化活性起着重要的作用. 该课题组通过 进一步的实验证实金-钯合金在可见光照射下能够驱动 芳香醇 $\alpha-\mathrm{H}$ 的离去, 从而使得该氧化反应无需在高温、 高氧条件下进行 ${ }^{[12]}$. 然而, 对于 $\mathrm{Au}-\mathrm{Cu} / \mathrm{TiO}_{2}$ 催化剂, 由 于 $\mathrm{Au}$ 的等离子活性使得表面被氧气氧化的铜得以还原, 从而保持合金的催化效果，促进醇类氧化反应进行. 等 离子体金纳米表面 $\mathrm{Cu}^{0}$ 的氧化与氧化后再还原为 $\mathrm{Cu}^{0}$ 会 加速电子转移, 从而促进整个氧化过程.

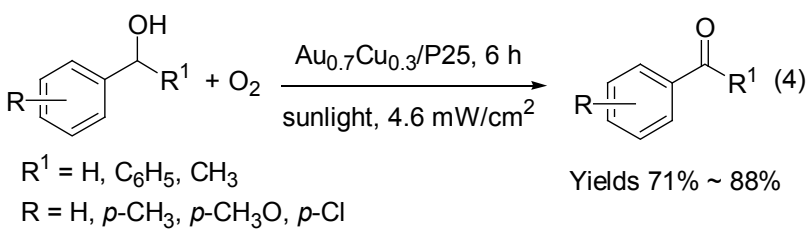

最近 Yang 等 ${ }^{[14]}$ 将 $\mathrm{Ag}$ 负载到 $\mathrm{P} 25 \mathrm{TiO}_{2}$ 和 $\mathrm{SiO}_{2}$ 上, 分 别制备了 $\mathrm{Ag} / \mathrm{TiO}_{2}$ 和 $\mathrm{Ag} / \mathrm{SiO}_{2}$ 光催化剂, 在紫外光照射 下用于催化甲醇氧化生成甲酸甲酯的反应. 实验发现两 种催化剂都表现出较高的催化活性. 其中 $\mathrm{Ag} / \mathrm{TiO}_{2}$ 催化 活性是由于 $\mathrm{TiO}_{2}$ 表面的 $\mathrm{Ag}$ 纳米粒子可以捕获 $\mathrm{TiO}_{2}$ 表 面上的光激发产生的电子，从而延长了电子和空穴的寿 命; $\mathrm{Ag} / \mathrm{SiO}_{2}$ 的催化作用主要来自于 $\mathrm{Ag}$ 纳米粒子的表面 等离子共振效应.

\section{2 烯烃的环氧化反应}

除了金以外，银、铜纳米粒子也可以被用作可见光 等离子光催化剂进行选择性环氧化反应. Linic 等 ${ }^{[15]}$ 的 研究证实负载在 $\alpha-\mathrm{Al}_{2} \mathrm{O}_{3}$ 上的边长为 $60 \mathrm{~nm}$ 的银立方体 可以催化工业生产中选择性氧化乙烯为环氧乙烷的重 要反应 $\mathrm{Ag} / \alpha-\mathrm{Al}_{2} \mathrm{O}_{3}$ (Eq. 5). 在 $450 \mathrm{~K}$, 可见光照射下氧 化速率比只有热驱动的体系提高 4 倍. 然而, 反应速率 的增高依据温度变化而不同, 从低温时 8 倍减小到高温 时的 3 倍, 但并没有影响环氧乙烷的选择性氧化. 通过 同位素标记氧原子 ${ }^{18} \mathrm{O}_{2}$ 的动力学研究, 发现光催化过程 具有较大的动力学同位素效应(KIEs) $\left({ }^{16} \mathrm{O} /{ }^{18} \mathrm{O}\right)$, KIE 值 约为 $1.19 \pm 0.01$, 说明 $\mathrm{O}_{2}$ 的活性是控制环氧化反应速率 的重要因素, 除了大的 KIE 值, 反应速率随光照的强度 的增加而提高, 这些进一步说明高能量电子可加速 $\mathrm{O}_{2}$ 分解过程, 进而驱动反应的快速进行.

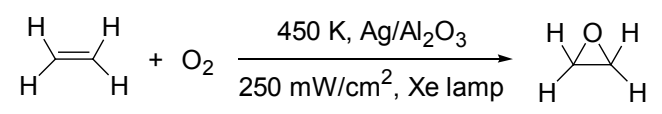


$\mathrm{Cu}$ 也可以作为一些反应的催化剂, 但是因为容易 被氧化, 很少被用于催化烯烃的环氧化反应. Linic 等 ${ }^{[16]}$ 将平均颗粒大小为 $(41 \pm 9) \mathrm{nm}$ 的 $\mathrm{Cu}$ 粒子附着在 $\mathrm{SiO}_{2}$ 上 制成 $\mathrm{Cu} / \mathrm{SiO}_{2}$, 用于催化丙烯环氧化反应. 在可见光照 射下, $\mathrm{Cu} / \mathrm{SiO}_{2}$ 催化的反应的选择性大大提高, 其原因是 铜纳米粒子的局域表面等离子体共振效应削弱了 $\mathrm{Cu}-$ $\mathrm{O}$ 键，因此促进了 $\mathrm{Cu}_{2} \mathrm{O}$ 还原成 $\mathrm{Cu}^{0}$.

\section{3 苯羟基化反应}

苯环中 $\mathrm{sp}^{2}$ 杂化的 $\mathrm{C}-\mathrm{H}$ 键比 $\mathrm{sp}^{3}$ 杂化的 $\mathrm{C}-\mathrm{H}$ 更稳 定, 这使得苯羟基化非常困难. 等离子体光催化剂在紫 外或可见光的照射下可催化此类反应. Ide 等 ${ }^{[17]}$ 将 $\mathrm{Au} / \mathrm{TiO}_{2}$ 成功地用于催化苯的羟基化反应(Eq. 6). $\mathrm{Au} / \mathrm{TiO}_{2}$ 是通过在无水乙醇中 $\mathrm{TiO}_{2}$ 和 $\mathrm{HAuCl}_{4}$ 室温反应 $1 \mathrm{~h}$, 然后用 $\mathrm{NaBH}_{4}$ 还原制备的. 在模拟的太阳光(含紫 外光)照射下, 苯发生羟基化反应生成苯酚. 当反应体 系中引入 $\mathrm{CO}_{2}, \mathrm{CO}_{2}$ 压力为 $230 \mathrm{kPa}$ 时, $\mathrm{Au} / \mathrm{TiO}_{2}$ 催化该反 应的选择性可达 $95 \%$, 苯的转化率为 $14 \%$; 无 $\mathrm{CO}_{2}$ 时反 应的选择性只有 $72 \%$.

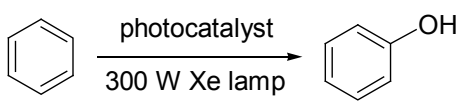

\footnotetext{
$\mathrm{Au} / \mathrm{TiO}_{2}, \mathrm{CO}_{2}$ : conv. $14 \%$, select. $95 \%$ Au/titanate, $\mathrm{O}_{2}$ : conv. $64 \%$, select. $96 \%$ $\mathrm{Au} / \mathrm{TiO}_{2}, \mathrm{O}_{2}$ : conv. $69 \%$, select. $91 \%$
}

Ogawa 等 ${ }^{[18]}$ 将金纳米负载于层状 $\mathrm{TiO}_{2}$ 夹层之间得 到盘状结构的金纳米盘, 同样可以催化可见光引发的苯 差基化反应. 该催化剂的制备过程是先用(3-颈基丙基) 三甲氧基硅烷修饰 $\mathrm{TiO}_{2}$, 硫醇修饰的 $\mathrm{TiO}_{2}$ 再和 $\mathrm{HAuCl}_{4}$ 混合, 随后用 $\mathrm{NaBH}_{4}$ 还原. 该催化剂在可见光的照射 下，对苯酚选择性高达 $96 \%$ (Eq. 6). 值得一提的是反应 开始时在体系中添加苯酚可以提高反应速率及产物的 选择性.

Huang 等 ${ }^{[19]}$ 用简便方法将贵金属负载到 $\mathrm{TiO}_{2}$ 上制 备了贵金属等离子体催化剂 $\mathrm{M} @ \mathrm{TiO}_{2}(\mathrm{M}=\mathrm{Au}, \mathrm{Pt}, \mathrm{Ag})$. 即紫外光照射下, 将 $\mathrm{TiO}_{2}$ 粉分散到无水乙醇中, 于是在 $\mathrm{TiO}_{2}$ 表面会产生 $\mathrm{Ti}^{3+}$, 然后在暗处加入贵金属盐, $\mathrm{Ti}^{3+}$ 就会还原金属阳离子使贵金属原位沉积在 $\mathrm{TiO}_{2}$ 表面. 这种制备方法会使贵金属纳米粒子均匀负载于 $\mathrm{TiO}_{2}$ 表 面, 使光催化反应在 $\mathrm{TiO}_{2}$ 表面发生. 所合成的三种催化 剂中, $\mathrm{Au} / \mathrm{TiO}_{2}$ 催化活性最高, 是水相中氧气氧化使苯发 生羟基化反应的一种有效的可见光响应光催化剂, 反应 的选择性可达 $91 \%$, 苯酚产率为 $63 \%$.

\section{4 硝基化合物的还原}

$\mathrm{Au} / \mathrm{ZrO}_{2}$ 在可见光的照射下催化醇氧化反应活性较 差. 最近澳大利亚昆士兰科技大学朱怀勇教授 ${ }^{[20]}$ 报道 了在紫外灯或白炽灯的照射下, $\mathrm{Au} / \mathrm{ZrO}_{2}$ 催化硝基化合
物选择性还原为相应的偶氮化合物的反应. 反应以异丙 醇为溶剂, 并添加了适量 $\mathrm{KOH}$, 且通入 $\mathrm{Ar}$ 气条件下进 行(Eq. 7). 将 $\mathrm{ZrO}_{2}$ 分散到 $\mathrm{HAuCl}_{4}$ 水溶液中, 加入赖氨 酸剧烈摚拌 $30 \mathrm{~min}$, 再慢慢加入 $\mathrm{NaBH}_{4}$ 还原, 最后加入 盐酸, 混合液静置 $24 \mathrm{~h}$, 即可得到固体 $\mathrm{Au} / \mathrm{ZrO}_{2}$ 催化剂, 金纳米粒子的大小约为 $6 \mathrm{~nm}$. 当光波长为 $400 \mathrm{~nm}$, 光强 度为 $0.30 \mathrm{~W} \cdot \mathrm{cm}^{-2}$ 时该催化剂可高效促进硝基苯还原为 偶氮苯，且反应具有高选择性. 产物除了芳香族偶氮类 化合物和丙酮外，该光催化过程中还产生了副产物 $\mathrm{O}_{2}$. 这一发现将加深我们对由等离子光催化剂引发的人工 光合作用使水分解产生氧气的理解. 实验对 $\mathrm{Au} / \mathrm{ZrO}_{2}$ 催 化硝基苯还原反应的的机理进行了探讨，认为在催化剂 表面形成的 $\mathrm{Au}-\mathrm{H}$ 物种在光催化反应中起着关键的作用. 在还原过程中的关键步骤是 $\mathrm{Au}-\mathrm{H}$ 物种使得 $\mathrm{N}-\mathrm{O}$ 键发 生断裂, $\mathrm{Au}-\mathrm{H}$ 与 $\mathrm{N}-\mathrm{O}$ 发生反应生成 HO-AuNP 物种, 该 物种很快就会分解产生氧气并再生 $\mathrm{Au}-\mathrm{H}$ 物种. 反应中 被光照射后金纳米粒子产生高能电子，亲电的 $\mathrm{N}-\mathrm{O}$ 键 的断裂易于在高能电子存在的条件下进行. 由于 $\mathrm{ZrO}_{2}$ 的导带较高, 因此电子不能从金纳米粒子转移到 $\mathrm{ZrO}_{2}$ 中, $\mathrm{ZrO}_{2}$ 在此光催化过程中只是作为金纳米粒子的载 体, 不能起到氧化还原作用.

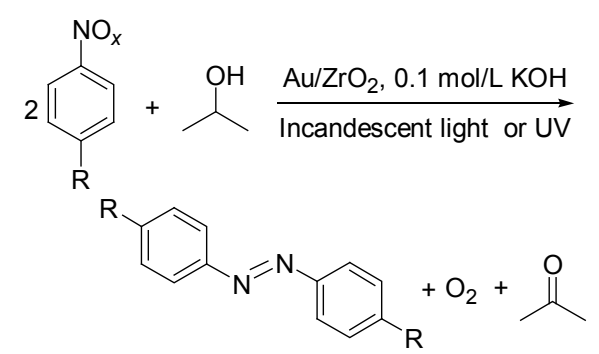

$x=2, \mathrm{R}=\mathrm{H}, \mathrm{Cl}, \mathrm{CH}_{3}, \mathrm{CH}_{3} \mathrm{O}$ : conv. $58 \% \sim 100 \%$, select. $56 \% \sim 99 \%$ $x=1, \mathrm{R}=\mathrm{H}$ : conv. $100 \%$, select. $95 \%$

银作为表面等离子体光催化剂，也可催化还原硝基 芳烃为偶氮类化合物. 将银涂抹在原子力显微镜针尖上 作催化剂, 在 $532 \mathrm{~nm}$ 激光照射下催化还原对硝基苯硫 酚为偶氮类化合物，其反应机制及分子动力学研究通过 双波长拉曼光谱进行监测 ${ }^{[21]}$, 详细的分子反应过程, 如 单层无序的反应及单一催化剂位点上的反应可以通过 此方法直接观察到. 另外, Kominami 等 ${ }^{[22]}$ 以 $\mathrm{Ag}$ 纳米粒 子作为一种助催化剂, 将等离子体光催化剂 $\mathrm{Au} / \mathrm{TiO}_{2}$ 进 行功能化, 制备了新型催化剂 $\mathrm{Au} / \mathrm{TiO}_{2}-\mathrm{Ag}$, 在可见光照 射下在异丙醇溶剂中催化还原硝基苯为苯胺. 实验表明 该催化剂比 $\mathrm{Au} / \mathrm{TiO}_{2}$ 显示更高的催化活性, 可以催化硝 基苯定量还原为苯胺.

虽然铜纳米和金、银纳米一样，在可见光区表现出 强的等离子共振效应，但其用作光催化剂却很少报道， 原因在于铜纳米在空气中或微量分子氧存在下很容易 
被氧化成氧化铜或氧化亚铜. 石墨烯(graphene)上的碳 空位和悬键可影响铜原子的电子结构, 从而利于提高其 稳定性. 山西煤化所郭向云课题组 ${ }^{[23]}$ 成功将铜纳米粒 子负载到石墨烯上制备了新型等离子体光催化剂 $\mathrm{Cu} / \mathrm{graphene}$, 该催化剂利用铜纳米粒子的局域表面等 离子体共振效应, 在可见光照射下可有效地催化芳香族 硝基化合物转化为相应的偶氮和氧化偶氮化合物(Eq. 8). 硝基苯和对硝基甲苯得到偶氮化合物的选择性分别 为 $98.2 \%$ 和 $95.8 \%$, 而对氯硝基苯、对硝基苯甲醚、对 硝基苯乙酮得到偶氮化合物的选择性在 45.9\%和 59.4\% 之间，此外还得到氧化偶氮化合物.

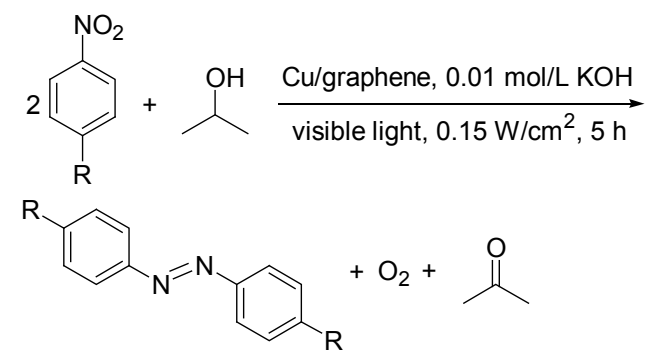

$\mathrm{R}=\mathrm{H}, p-\mathrm{Cl}, p-\mathrm{CH}_{3}, p-\mathrm{CH}_{3} \mathrm{O}, p-\mathrm{CH}_{3} \mathrm{CO}$

conv. $66.3 \% \sim 97.9 \%$, select. $45.9 \% \sim 98.2 \%$

\section{5 胺的氧化}

亚胺类物质是制备染料、药物等精细化工产品中经 常使用的一类中间体, 可以通过胺的氧化来制备. $\mathrm{Au}-\mathrm{Pd}$ 合金负载在 $\mathrm{ZrO}_{2}$ 上制成 $\mathrm{Au}-\mathrm{Pd} @ \mathrm{ZrO}_{2}{ }^{[11]}$ 或者粒径 为 $7 \mathrm{~nm}$ 的金纳米负载在金红石 $\mathrm{TiO}_{2}$ 上制成 $\mathrm{Au} / \mathrm{TiO}_{2}{ }^{[24]}$ 均可以作为等离子光催化剂, 用于选择性氧化芳香胺类 物质. 在 $\mathrm{CH}_{3} \mathrm{CN}$ 介质中, $\mathrm{Au}-\mathrm{Pd} @ \mathrm{ZrO}_{2}$ 可选择氧化苯甲 胺为亚胺, 产率最高达 $95 \%$ (Eq. 9). 在可见光的照射下, 无溶剂条件下 $\mathrm{Au} / \mathrm{TiO}_{2}$ 既能氧化苯甲胺为亚胺(Eq. 9), 也能成功地催化苯甲基仲胺氧化为亚胺. 许多二价或三 价金属氧化物包括锐铁矿 $\mathrm{TiO}_{2}$ 、金红石 $\mathrm{TiO}_{2} 、 \mathrm{Nb}_{2} \mathrm{O}_{5}$ 、 $\mathrm{ZnO} 、 \mathrm{WO}_{3} 、 \mathrm{In}_{2} \mathrm{O}_{3}$ 和 $\mathrm{SrTiO}_{3}$ 被用作载体, 且金红石的 $\mathrm{TiO}_{2}$ 表现出最好的催化结果, 并且选择性高于 $99 \%$, 然 而, 反应转化率非常低 $(<5 \%)$.

$$
\mathrm{R}=\mathrm{H}, p-\mathrm{Cl}, p-\mathrm{CH}_{3}, p-\mathrm{CH}_{3} \mathrm{O}
$$

Au-Pd/ZrO $2,>400 \mathrm{~nm}$ : Yields 33\% 95\%

Au/rutile $\mathrm{TiO}_{2},>430 \mathrm{~nm}$ : conv. $0.7 \% \sim 4.5 \%$, select. $99 \%$

\section{6 炔的氢胺化反应}

氢胺化反应是一类符合原子经济性和绿色化学的 反应, 是一类区域选择性地形成碳氮键的重要方法, 由
于含氮化合物几乎涵盖了大部分的医药化合物及很多 具有生物活性的有机分子, 因此该反应无论在学术科研 领域上还是在工业生产都引起了广泛关注. 朱怀勇 等 ${ }^{[25]}$ 将金负载在 $\mathrm{N}$ 掺杂的 $\mathrm{TiO}_{2}$ 上的制备成 $\mathrm{Au} / \mathrm{TiO}_{2}-\mathrm{N}$, 以此作为等离子体光催化剂催化炔烃和芳香胺的氢胺 化反应，反应具有选择性好、产率高的特点(Eq. 10). 实 验考察了不同载体的催化效果, 发现以 $\mathrm{N}$ 掺杂的 $\mathrm{TiO}_{2}$ 为载体负载金属纳米粒子比其他载体的效果好, 因掺杂 氮使 $\mathrm{TiO}_{2}$ 中含有 $\mathrm{Ti}^{3+}$, $\mathrm{Ti}^{3+}$ 可以为炔烃提供更多的反应 位点, 因此, 比纯的 $\mathrm{TiO}_{2}$ 效果好. 结果表明金纳米粒子 和无机载体对活化反应物都起着重要作用.

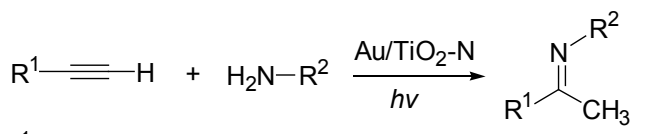

$$
\begin{aligned}
& \mathrm{R}^{1}=\mathrm{Ph}, \mathrm{BrC}_{6} \mathrm{H}_{4}, \mathrm{CH}_{3} \mathrm{C}_{6} \mathrm{H}_{4}, \mathrm{CH}_{3} \mathrm{OC}_{6} \mathrm{H}_{4} \text {, conv. } 0.5 \% \sim 93 \% \\
& \mathrm{PhCH}_{2}, \mathrm{PhCH}_{2} \mathrm{CH}_{2}, \mathrm{CH}_{3}\left(\mathrm{CH}_{2}\right)_{5} \quad \text { select. } 50 \% \sim 99 \% \\
& \mathrm{R}^{2}=\mathrm{Ph}, \mathrm{BrC}_{6} \mathrm{H}_{4}, \mathrm{ClC}_{6} \mathrm{H}_{4}, \mathrm{CH}_{3} \mathrm{C}_{6} \mathrm{H}_{4}, \mathrm{CH}_{3} \mathrm{OC}_{6} \mathrm{H}_{4}, \mathrm{C}_{6} \mathrm{H}_{11}
\end{aligned}
$$

\section{7 炔丙胺的合成}

Gonzalez-Bejar 等 ${ }^{[26]}$ 将 $\mathrm{Au} / \mathrm{ZnO}$ 作为催化剂, 用于 催化多组分缩合反应, 在 $530 \mathrm{~nm}$ 光照射下, 将醛、胺、 炔烃混合，室温反应 $2 \mathrm{~h}$ 即可一锅法合成炔丙胺(Eq. 11). 催化剂中除了金纳米粒子的催化活性外, 载体 $\mathrm{ZnO}$ 和 炔烃的相互作用也有助于提高反应产率.
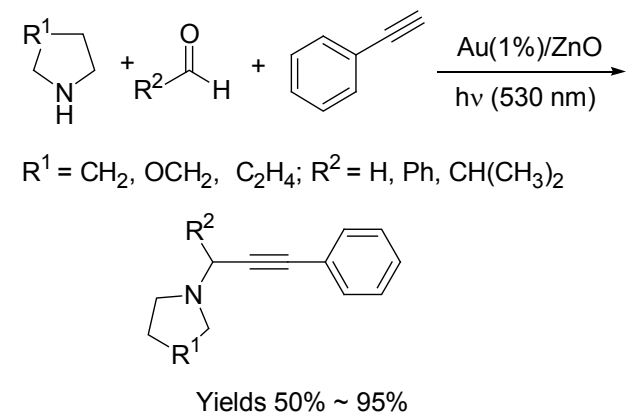

\section{8 胺的氧化缩合反应}

吗啉衍生物具有重要的药理活性, 在可见光的照射 下, 等离子光催化剂可用于催化醛和吗啉的氧化缩合反 应. Luque 研究组 ${ }^{[27]}$ 发现, 在双氧水和强碱 $\mathrm{KOH}$ 存在下, 以 $\mathrm{Au} / \mathrm{SiO}_{2}$ 为光催化剂, $532 \mathrm{~nm}$ 激光照射下可催化吗啉 和苯甲醛反应合成酰胺类化合物(Eq. 12). 因为双氧水 可以选择性氧化苯甲醇为苯甲醛，所以也可以用芳香醇 作为合成该类酰胺的起始底物.

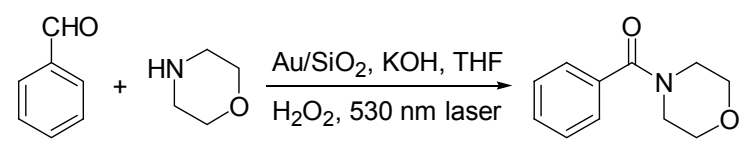




\subsection{Suzuki 偶联反应}

碳-碳偶联反应是构建 $\mathrm{C}-\mathrm{C}$ 键最有效的方法, 目 前已广泛应用于天然产物、医药、农药、以及功能材料 等的合成. 北京大学严纯华研究组 ${ }^{[28]}$ 发现采用异质外 延生长法制备的 Au-Pd 纳米材料可以吸收可见光至近 红外光区域范围内的光波, 在 $809 \mathrm{~nm}$ 激光的照射下, $\mathrm{Au}-\mathrm{Pd}$ 纳米材料可作为苯硼酸和澳苯进行 Suzuki 偶联反 应的有效光催化剂(Eq. 13). 其中等离子体金纳米核能 有效吸收可见光, 而钯纳米粒子作为直接的催化剂催化 偶联反应. 实验证实等离子光催化作用和光热效应都对 催化活性起重要作用. 实验探讨了 5 种不同的 $\mathrm{Au}-\mathrm{Pd}$ 的 纳米材料(4 种不同规格的纳米棒, 1 种纳米球)的催化效 率, 发现催化效率的大小取决于 $\mathrm{Au}-\mathrm{Pd}$ 纳米棒的大小, 具有更小尺寸的的 Au-Pd 纳米棒表现出更好催化性能. $\mathrm{Au}-\mathrm{Pd}$ 纳米棒的催化活性与等离子共振激发产生的热电 子有关, 用 $\mathrm{TiO}_{2}$ 壳阻断 $\mathrm{Au} 、 \mathrm{Pd}$ 间的光生电子转移, 其 催化活性显著降低. 单独的金、钯纳米和金钯纳米的混 合物对该反应均无光催化活性.

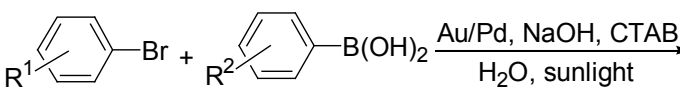

$\mathrm{R}^{1}=\mathrm{H}, o-\mathrm{CH}_{3} \mathrm{O}, p-\mathrm{CH}_{3} \mathrm{O}, m-\mathrm{CH}_{3} \mathrm{O}$

$\mathrm{R}^{2}=\mathrm{H}, o-\mathrm{CH}_{3}, p-\mathrm{CH}_{3}, m-\mathrm{CH}_{3}$

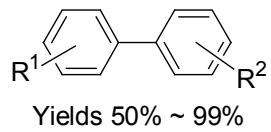

以 $\mathrm{Au}-\mathrm{Pd} @ \mathrm{ZrO}_{2}$ 为催化剂, 在可见光的照射下，可 以有效催化碘苯和苯硼酸的 Suzuki 偶联反应(Eq. 14) ${ }^{[11]}$. 合金组成对其光催化效率有显著影响, 金、钯物质的量 比为 $1: 1.86$ 时光催化活性最高, 其他比例时催化活性 显著降低, 这与合金表面电荷的非均一程度有关. 其催 化机理为由于金纳米的表面等离子体共振作用，在钯位 点表面产生高能电子, 提高了钯固有的催化活性, 从而 加速了偶联反应.

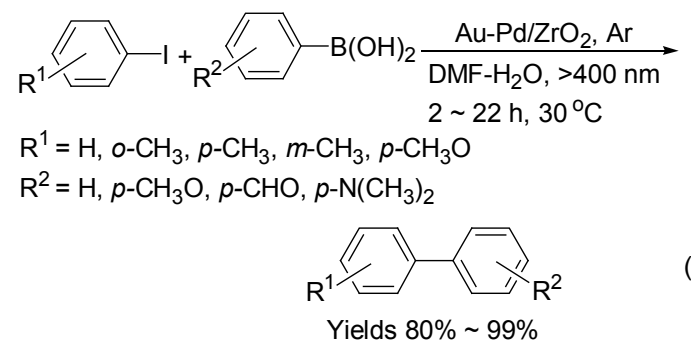

Huang 等 ${ }^{[29]}$ 合成了独特的双金属轮状结构的等离 子体光催化剂 $\mathrm{Au}-\mathrm{Pd}$ 纳米轮, 在可见光照射下成功地用 于催化碘苯和苯硼酸的 Suzuki 偶联反应，50 ${ }^{\circ} \mathrm{C}$ 光照 90 min 可定量得到联苯，反应速率是同温度下暗处反应的 3.8 倍. 单独的金、钯纳米和金钯纳米的混合物对该反应 基本均无光催化活性，这表明 $\mathrm{Au}-\mathrm{Pd}$ 纳米轮独特的异质 结结构使该催化剂显示出较高的催化活性.

我们课题组 ${ }^{[30]}$ 研究发现等离子光催化剂 $\mathrm{Ag}-\mathrm{AgBr}$ 经氧化石墨烯-Pd 功能化后得到的 GO-Pd/Ag-AgBr, 在 可见光辐射下可高效率催化溴苯和苯硼酸的 Suzuki 偶 联反应(Eq. 15). 结果表明, 氧化石墨烯的存在利于光生 电子和空穴的分离，反应体系中加入空穴捕获剂三乙醇 胺, 产物产率显著降低, 表明空穴在活化底物中发挥关 键作用; 光生高能电子转移到钯纳米表面，提高了其电 子密度, 从而利于其活化碳卤键. 将光催化剂与具有内 在催化活性的金属相结合用于光催化有机反应为设计 功能化等离子体光催化剂提供了一条新思路.

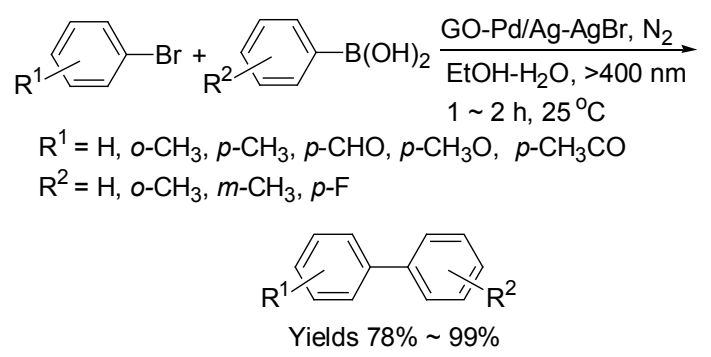

\subsection{0 氯苯脱氯反应}

氯代有机物是重要的化工原料，由于其致癌作用， 且在环境中难以降解, 对环境造成了巨大的危害, 因此, 氯代有机物的脱氯成为了消除有机氯污染的有效方法. Kominami 等 ${ }^{[31]}$ 采用简单的两步光化学沉积方法合成了 核壳结构的 $\mathrm{Au} @ \mathrm{Pd} / \mathrm{TiO}_{2}$ 等离子体光催化剂, 在异丙 醇一水介质中，可见光照射下室温反应 $20 \mathrm{~h}$ ，该催化剂 可催化氯苯脱氯定量转化为苯, 同时异丙醇被氧化转化 为丙酮(Eq. 16). 结果表明钯壳厚度对催化效果及光的 吸收都有显著影响, 钯壳厚度为 $1 \mathrm{~nm}$ 时, 即 $\mathrm{Au}(0.8) @ \mathrm{Pd}(0.2) / \mathrm{TiO}_{2}$, 催化效果最好, 其在 $550 \mathrm{~nm}$ 有 强的可见光吸收. 而 $\mathrm{Au} / \mathrm{TiO}_{2}$ 和 $\mathrm{Pd} / \mathrm{TiO}_{2}$ 均无催化活性. 在等离子体光催化剂 $\mathrm{Au} / \mathrm{TiO}_{2}$ 中引入共催化剂 $\mathrm{Pd}$ 提高 了 $\mathrm{Au} / \mathrm{TiO}_{2}$ 对卤代烃脱氯反应的催化活性. 作者提出的 可能催化机理为: 经表面等离子共振激发在金表面产生 高能电子, 电子随即转移到钯壳表面, 氯苯从钯壳上获 得电子转化为苯; 产生的缺电子的金纳米从电子供体异 丙醇获得电子恢复其金属态，同时异丙醇转化为丙酮.

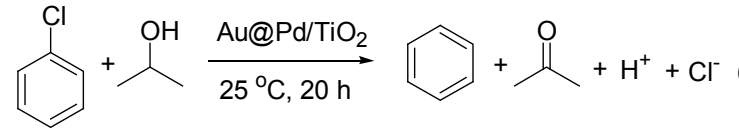




\section{3 结论与展望}

总之, 光催化选择性有机合成作为光催化领域的一 个前沿方向, 越来越受到人们的重视. 等离子体光催化 剂作为一类新型可见光响应的光催化剂, 其表面等离子 体金属 $(\mathrm{Au}, \mathrm{Ag}, \mathrm{Cu})$ 能够更加高效的吸收可见光产生高 能电子进而引发有机合成反应, 目前已成功用于催化醇 氧化、烯烃环氧化、硝基还原、碳碳偶联、苯着基化等 诸多重要的有机反应, 从而展示出光催化技术作为一项 绿色有机合成途径所具有的巨大潜力, 这些开创性的基 础研究工作为有效利用太阳能作为化学反应的新能源 开辟了一条新路. 但等离子体光催化剂在有机合成中的 应用仍然面临诸多问题有待解决, 如光的量子产率不 高, 因此开发高效催化剂, 提高光的利用率是当前的迫 切任务; 催化剂多利用贵金属 $\mathrm{Au}, \mathrm{Ag}$ 的等离子共振效 应, 成本较高, 且多为纳米颗粒, 回收困难, 同时容易 造成流失浪费, 如何降低催化剂成本、实现催化剂的固 定化是需要解决的一个问题; 等离子体光催化合成机理 的研究也是摆在科学工作者面前的紧要课题, 很多反应 条件对实验结果的影响尚不明确, 如何设计一个简单模 型，探索光催化有机合成的最基本原理和普遍规律，为 研制高活性和高选择性的光催化剂提供理论指导, 是未 来研究者需考虑的一个问题; 合理的光催化反应器设计 也是未来研究的一个重要方向.

尽管光催化有机合成走向实际应用, 仍然面临巨大 的挑战, 但是从节能和环保的长远角度看, 光催化有机 转化必将成为 21 世纪最具潜力的、高效、绿色的有机 合成技术之一。

\section{References}

[1] (a) Chen, C.; Ma, W.; Zhao, J. Chem. Soc. Rev. 2010, 39, 4206 (b) Tong, H.; Ouyang, S.; Bi, Y.; Umezawa, N.; Oshikiri, M.; Ye, J. Adv. Mater. 2012, 24, 229.

(c) Xiang, Q.; Yu, J.; Jaroniec, M. Chem. Soc. Rev. 2012, 41, 782.

[2] (a) Colmenares, J. C.; Luque, R. Chem. Soc. Rev. 2014, 43, 765. (b) Sarina, S.; Waclawik, E. R.; Zhu, H. Green Chem. 2013, 15, 1814 .

[3] (a) Lang, X.; Chen, X.; Zhao, J. Chem. Soc. Rev. 2014, 43, 473. (b) Zhang, Y.; Zhang, N.; Tang, Z.-R.; Xu, Y.-J. ACS Sustainable Chem. Eng. 2013, 1, 1258 .

[4] (a) Linic, S.; Christopher, P.; Ingram, D. B. Nat. Mater. 2011, 10, 911.

(b) Hou, W.; Cronin, S. B. Adv. Funct. Mater. 2013, 23, 1612.
[5] Tsukamoto, D.; Shiraishi, Y.; Sugano, Y.; Ichikawa, S.; Tanaka, S.; Hirai, T. J. Am. Chem. Soc. 2012, 134, 6309.

[6] Naya, S.-I.; Inoue, A.; Tada, H. J. Am. Chem. Soc. 2010, 132, 6292.

[7] Tanaka, A.; Hashimoto, K.; Kominami, H. Chem. Commun. 2011, 47,10446

[8] Tanaka, A.; Hashimoto, K.; Kominami, H. J. Am. Chem. Soc. 2012, $134,14526$.

[9] Maldotti, A.; Molinari, A.; Juárez, R.; Garcia, H. Chem. Sci. 2011, 2,1831 .

[10] Hallett-Tapley, G. L.; Silvero, M. J. N.; González-Béjar, M. A.; Grenier, M.; Netto-Ferreira, J. C.; Scaiano, J. C. J. Phys. Chem. C 2011, 115, 10784.

[11] Sarina, S.; Zhu, H.; Jaatinen, E.; Xiao, Q.; Liu, H.; Jia, J.; Chen, C.; Zhao, J. J. Am. Chem. Soc. 2013, 135, 5793.

[12] Sarina, S.; Bai, S.; Huang, Y.; Chen, C.; Jia, J.; Jaatinen, E.; A. Ayoko, G.; Bao, Z.; Zhu, H. Green Chem. 2014, 16, 331.

[13] Sugano, Y.; Shiraishi, Y.; Tsukamoto, D.; Ichikawa, S.; Tanaka, S.; Hirai, T. Angew. Chem., Int. Ed. 2013, 52, 5295.

[14] Yang, X.; Zhang, A.; Gao, G.; Han, D.; Han, C.; Wang, J.; Lu, H.; Liu, J.; Tong, M. Catal. Commun. 2014, 43, 192.

[15] Christopher, P.; Xin, H.; Linic, S. Nat. Chem. 2011, 3, 467.

[16] Marimuthu, A.; Zhang, J.; Linic, S. Science 2013, 339, 1590.

[17] Ide, Y.; Nakamura, N.; Hattori, H.; Ogino, R.; Ogawa, M.; Sadakane, M.; Sano, T. Chem. Commun. 2011, 47, 11531.

[18] Ide, Y.; Matsuoka, M.; Ogawa, M. J. Am. Chem. Soc. 2010, 132, 16762.

[19] Zheng, Z.; Huang, B.; Qin, X.; Zhang, X.; Dai, Y.; Whangbo, M.-H. J. Mater. Chem. 2011, 21, 9079.

[20] Zhu, H.; Ke, X.; Yang, X.; Sarina, S.; Liu, H. Angew. Chem., Int. Ed. 2010, 49, 9657.

[21] van Schrojenstein Lantman, E. M.; Deckert-Gaudig, T.; Mank, A. J.; Deckert, V.; Weckhuysen, B. M. Nat. Nanotechnol. 2012, 7, 583.

[22] Tanaka, A.; Nishino, Y.; Sakaguchi, S.; Yoshikawa, T.; Imamura, K.; Hashimoto, K.; Kominami, H. Chem. Commun. 2013, 49, 2551.

[23] Guo, X.; Hao, C.; Jin, G.; Zhu, H.-Y.; Guo, X.-Y. Angew. Chem., Int. Ed. 2014, 126, 2004.

[24] Naya, S.-I.; Kimura, K.; Tada, H. ACS Catal. 2013, 3, 10.

[25] Zhao, J.; Zheng, Z.; Bottle, S.; Chou, A.; Sarina, S.; Zhu, H. Chem. Commun. 2013, 49, 2676.

[26] Gonzalez-Bejar, M.; Peters, K.; Hallett-Tapley, G. L.; Grenier, M.; Scaiano, J. C. Chem. Commun. 2013, 49, 1732.

[27] Pineda, A.; Gomez, L.; Balu, A. M.; Sebastian, V.; Ojeda, M.; Arruebo, M.; Romero, A. A.; Santamaria, J.; Luque, R. Green Chem. 2013, 15, 2043.

[28] Wang, F.; Li, C.; Chen, H.; Jiang, R.; Sun, L. D.; Li, Q.; Wang, J.; Yu, J. C.; Yan, C. H. J. Am. Chem. Soc. 2013, 135, 5588.

[29] Huang, X.; Li, Y.; Chen, Y.; Zhou, H.; Duan, X.; Huang, Y. Angew. Chem., Int. Ed. 2013, 52, 6063.

[30] Gao, S. T.; Shang, N. Z., Feng, C.; Wang, C.; Wang, Z. RSC Adv. 2014, 4, 39242.

[31] Tanaka, A.; Fuku, K.; Nishi, T.; Hashimoto, K.; Kominami, H. J. Phys. Chem. C 2013, 117, 16983. 